\title{
Adrenal crisis and acute exacerbation of interstitial lung disease after thymoma needle biopsy: a case report and literature review
}

\author{
Hengyi Yan ${ }^{1}$, Qi Qi ${ }^{1}$, Kai Zhang ${ }^{2}$, Bing $\mathrm{He}^{3}$, Guoming Chu ${ }^{4}$, Guan Wang ${ }^{5}$, Hua Wen ${ }^{6}$, Yanyan Zhao ${ }^{4}$, \\ Hongxu Liu ${ }^{5}$, Li Zhao', Yu Chen ${ }^{1}$ \\ ${ }^{1}$ Department of Pulmonary and Critical Care Medicine, Shengjing Hospital of China Medical University, Shenyang, China; ${ }^{2}$ Department of \\ Gastroenterology, Endoscopic Center, Shengjing Hospital of China Medical University, Shenyang, China; ${ }^{3}$ The First Endocrinology Department, \\ Shengjing Hospital of China Medical University, Shenyang, China; ${ }^{4}$ Department of Clinical Genetics, Shengjing Hospital of China Medical \\ University, Shenyang, China; ${ }^{5}$ Department of Thoracic Surgery, Cancer Hospital of China Medical University, Liaoning Cancer Hospital \& \\ Institute, Shenyang, China; ${ }^{6}$ Department of Pulmonary and Critical Care Medicine, The First Affiliated Hospital of China Medical University, \\ Shenyang, China \\ Correspondence to: Yu Chen. Department of Pulmonary and Critical Care Medicine, Shengjing Hospital of China Medical University, No. 36 Sanhao \\ Street, Heping District, Shenyang 110004, Liaoning, China. Email: chenyusy@hotmail.com; Hongxu Liu. Department of Thoracic Surgery, \\ Cancer Hospital of China Medical University, Liaoning Cancer Hospital \& Institute, No. 44, Xiaoheyan Road, Dadong District, Shenyang 110042, \\ Liaoning, China. Email: hxliu@cmu.edu.cn; Yanyan Zhao. Department of Clinical Genetics, Shengjing Hospital of China Medical University, No. 36 \\ Sanhao Street, Heping District, Shenyang 110004, Liaoning, China. Email: yyzhao@sj-hospital.org.
}

\begin{abstract}
Thymoma is the most common paraneoplastic syndrome-associated tumor. It is related to a variety of autoimmune diseases including myasthenia gravis, systemic lupus erythematosus, and hypogammaglobulinemia. Only a few reports of thymoma associated with Addison's disease have been reported to date. Herein, we report a novel case of thymoma complicated with autoimmune Addison's disease and interstitial lung disease. The patient developed adrenal crisis with persistent hypotensive shock and heart block after needle biopsy. Acute exacerbation of the interstitial lung disease was also observed, accompanied by severe respiratory failure. After treatment with glucocorticoids, somatostatin, and temporary pacemaker implantation, the patient's condition improved, and the thymoma had shrunk in size. Finally, he underwent transsternal extended thymectomy and lymph node dissection. Hydrocortisone was given intravenously before surgery, on the operation day and after the surgery. The operation was uneventful, and no hypotension or fever occurred. Cortisol and ACTH were still obviously abnormal at 1 month post-surgery. The clinical manifestations of Addison's disease and interstitial lung disease are hidden and can be easily overlooked. However, in the postoperative period, Addison's disease can lead to adrenal crisis developing, which can progress to life-threatening shock, arrhythmia, and acute respiratory failure. Therefore, clinicians should be aware of this phenomenon and consider a regimen combining proactive glucocorticoid replacement therapy with somatostatin to preserve the life of such patients.
\end{abstract}

Keywords: Addison's disease; heart block; interstitial lung disease; shock; thymoma; case report

Submitted Mar 12, 2021. Accepted for publication May 20, 2021.

doi: $10.21037 /$ tlcr-21-195

View this article at: https://dx.doi.org/10.21037/tlcr-21-195

\section{Introduction}

Thymomas are rare solid tumors that are often accompanied by autoimmune diseases such as myasthenia gravis, hypogammaglobulinemia, and collagen vascular disease. Although a few cases of thymoma complicated with autoimmune Addison's disease have been documented in the literature (1-6), none of these patients had concomitant interstitial lung disease. Addison's disease has a long dormancy period, with clinical symptoms appearing only when more than $90 \%$ of both adrenal glands has been 
destroyed (7). The usual symptoms of this disorder are unexplained gastrointestinal distress, malaise, weight loss, hypoglycemia, and orthostatic hypotension. Typical manifestations include hyperpigmentation of the skin and mucous membranes. In patients with long-term untreated Addison's disease, stress conditions such as surgery, infection, and trauma can trigger the occurrence of lifethreatening shock and cardiac arrhythmias.

Interstitial lung disease, which may be caused by autoimmune diseases, describes a large group of disorders that result in progressive lung tissue scarring, which eventually affects the individual's ability to breathe. Herein, we report a novel case of thymoma complicated with Addison's disease and interstitial lung disease. The patient developed adrenal crisis after thymoma needle biopsy, which was accompanied by acute exacerbation of the interstitial lung disease and severe respiratory failure. Treatment with glucocorticoids, somatostatin, and temporary pacemaker implantation proved to be successful. Due to the complexity of the case and the patient's critical condition, a failure to diagnose and treat them immediately would have led to a poor prognosis. We present the following case in accordance with the CARE reporting checklist (available at https://dx.doi.org/10.21037/tlcr-21-195).

\section{Case presentation}

A 26-year-old male patient was admitted to the Department of Thoracic Surgery, Shengjing Hospital of China Medical University on March 25, 2020, with the chief complaints of intermittent cough, fatigue, and weight loss $(25 \mathrm{~kg}$ in the previous 6 months). A space-occupying lesion in the anterior mediastinum that had existed for 3 months, without a definitive diagnosis, was observed. The patient had no history of other disease and no family history of genetic disease. The vital signs, physical examination results, and laboratory findings on admission are shown in Figure 1. Enhanced computed tomography (CT) of the chest revealed a soft tissue mass with an unclear boundary and uneven enhancement in the anterior mediastinum, which was suspected as malignant, and mediastinal and hilar lymph node enlargement was also observed. Multiple nodules and a small number of ground-glass opacities were found in the lungs (Figure 2). Enhanced abdominal CT revealed slight enlargement of both the spleen and multiple lymph nodes in the retroperitoneum and right cardiophrenic angle region. No clear signs of bone metastasis were found on 99mTc-MDP whole-body bone scan, nor did head CT indicate any abnormalities. Lung function tests revealed a restrictive ventilatory defect: forced vital capacity (FVC): $2.03 \mathrm{~L}$; FVC\% pred: $42.5 \%$; forced expiratory volume in 1 second $\left(\mathrm{FEV}_{1}\right): 1.56 \mathrm{~L} ; \mathrm{FEV}_{1} \%$ pred: $38.5 \%$; $\mathrm{FEV}_{1} / \mathrm{FVC}$ : $77.09 \%$.

Based on these findings, the patient underwent CTguided needle biopsy of the anterior mediastinal mass on April 1, 2020. Four days later (April 5, 2020), the patient developed fever $\left(39^{\circ} \mathrm{C}\right.$ ) accompanied by dyspnea (Figure 3), which worsened on the following day. CT re-examination of the lung indicated that the ground-glass opacities had progressed significantly (Figure 2). On the evening of April 8, the patient's condition deteriorated with shock and respiratory failure (Figure 3), and he was subsequently transferred to the Department of Pulmonary and Critical Care Medicine, where he was immediately administered $40 \mathrm{mg}$ intravenous methylprednisolone, ertapenem and moxifloxacin as antibiotics, ganciclovir and umifenovir as antivirals, adequate fluid replacement $(2,885 \mathrm{~mL}$ over 8 hours), supportive dopamine hypertensive therapy, and high-flow oxygen inhalation. The patient's fever subsided the same evening that these treatments were received, and his respiratory failure was ameliorated; however, a 24-hour dopamine pump was still required to maintain blood pressure. Urine output was normal, but progressive exacerbated bradycardia developed (Table 1).

By April 12, oxygenation had been completely restored, so the patient's oxygen therapy was discontinued; however, continuous intravenous pumping of dopamine $(7 \mu \mathrm{g} / \mathrm{kg} / \mathrm{min})$ was still required to maintain blood pressure. On April 13, the methylprednisolone prescribed was reduced from $40 \mathrm{mg}$ every 12 hours to $40 \mathrm{mg}$ once a day. Continuous intravenous pumping of dopamine was increased to $9 \mu \mathrm{g} / \mathrm{kg} / \mathrm{min}$ plus $400 \mu \mathrm{g} / \mathrm{h}$ norepinephrine to maintain the mean arterial pressure at $54.7-82.7 \mathrm{mmHg}$. The patient's heart rate was 44 beats/min. Electrocardiogram showed sinus bradycardia, QT interval prolongation, and T-wave inversion (Figure 4). On April 14, the patient developed severe dyspnea accompanied by amaurosis. His heart rate dropped to 26 beats/min, and an electrocardiogram revealed an atrioventricular junctional escape rhythm and ventricular bigeminy (Figure 4). The patient was immediately administered $0.5 \mathrm{mg}$ atropine intravenously, and a temporary pacemaker was implanted. After the operation, somatostatin $(0.25 \mathrm{mg} / \mathrm{h})$ was administered via continuous intravenous infusion; one hour after the somatostatin administration, the mean arterial pressure increased from 58.7 to $96.7 \mathrm{mmHg}$. Methylprednisolone $(80 \mathrm{mg})$ was administered that night, 


\begin{tabular}{|c|c|c|c|c|c|}
\hline \multicolumn{6}{|l|}{ Vital signs } \\
\hline \multicolumn{6}{|c|}{ Physical examination on admission } \\
\hline \multicolumn{3}{|c|}{ Diffuse hyperpigmentation of the skin } & \multicolumn{3}{|c|}{ Pulmonary auscultation of bilateral small rales } \\
\hline \multicolumn{3}{|c|}{ Heart rhythm regular, with no murmurs } & \multicolumn{3}{|c|}{ Normal muscle strength in the extremities } \\
\hline \multicolumn{6}{|c|}{ No abnormalities indicated by nervous system examination } \\
\hline \multicolumn{6}{|c|}{ Laboratory findings } \\
\hline Peripheral blood & Value & Blood chemistry & Value & Arterial blood gas & Value \\
\hline WBC & $5.4 \times 10^{9} / \mathrm{L}$ & Total protein & $69.2 \mathrm{~g} / \mathrm{L}$ & $\mathrm{PH}$ & 7.396 \\
\hline Granulocytes & $58.2 \%$ & Albumin & $40.5 \mathrm{~g} / \mathrm{L}$ & $\mathrm{PCO}_{2}$ & $41.1 \mathrm{mmHg}$ \\
\hline Eosinophils & $0.9 \%$ & LDH & $237 \mathrm{U} / \mathrm{L}$ & & \\
\hline RBC & $4.1 \times 10^{12} / \mathrm{L}$ & Urea & $5 \mathrm{mmol} / \mathrm{L}$ & & \\
\hline Hemoglobin & $122 \mathrm{~g} / \mathrm{L}$ & Creatinine & 65.9 umol/L & & \\
\hline Platelets & $253 \times 10^{9} / \mathrm{L}$ & $\mathrm{CK}$ & $137 \mathrm{U} / \mathrm{L}$ & & \\
\hline \multicolumn{2}{|l|}{ Urine } & CK-MB & $11 \mathrm{U} / \mathrm{L}$ & & \\
\hline Specific gravity & 1.016 & $\mathrm{~K}$ & $4.59 \mathrm{mmol} / \mathrm{L}$ & & \\
\hline $\mathrm{Ph}$ & 5.0 & $\mathrm{Na}$ & 134 mmol/L & & \\
\hline Protein & $(-)$ & $\mathrm{Cl}$ & $99.2 \mathrm{mmol} / \mathrm{L}$ & & \\
\hline
\end{tabular}

Figure 1 Vital signs, physical examination results, and laboratory findings of the patient on admission. WBC, white blood cell count; RBC, red blood cell count; ALT, alanine aminotransferase; AST, aspartate aminotransferase; LDH, lactate dehydrogenase; CK, creatine kinase; $\mathrm{CK}-\mathrm{MB}$, creatine kinase $\mathrm{MB}$ isozymes; $\mathrm{K}$, serum potassium concentration; $\mathrm{Na}$, serum sodium concentration; $\mathrm{Cl}$, serum chlorine concentration; $\mathrm{Ca}$, serum calcium concentration; FBS, fasting blood glucose; ESR, erythrocyte sedimentation rate; $\mathrm{PCO}_{2}$, partial pressure of carbon dioxide; $\mathrm{PO}_{2}$, partial pressure of oxygen; $\mathrm{HCO}_{3}{ }^{-}$, bicarbonate.

and the highest mean arterial pressure rose to $103.3 \mathrm{mmHg}$; however, the blood pressure did not stabilize until after 10 days. The pathological report for the thymus biopsy indicated a type B2 thymoma (Figure 5).

A detailed review of the patient's medical history revealed that the patient experienced dizziness after standing for long periods, had low blood pressure, and had noticed a change in his skin pigmentation (Figure 6); because this strongly suggested that he might have Addison's disease, we performed an endocrine examination. The plasma cortisol (8 am; April 14) level was $0.161 \mu \mathrm{g} / \mathrm{dL}$ (normal: 6.02-18.4), which was remarkably low, while the plasma adrenocorticotropic hormone (ACTH, 8 am; April 14) level was $1,486 \mathrm{pg} / \mathrm{mL}$ (normal: $7.2-63.3$ ), which was remarkably high. The viral and bacterial infection indices were negative (Table 2). A diagnosis of Addison's disease was made on April 14, and paraneoplastic syndrome secondary to a thymoma was considered. The absence of antibodies against the acetylcholine receptor, muscle-specific kinase, doublestranded DNA, and Smith was noted. The oral glucose 

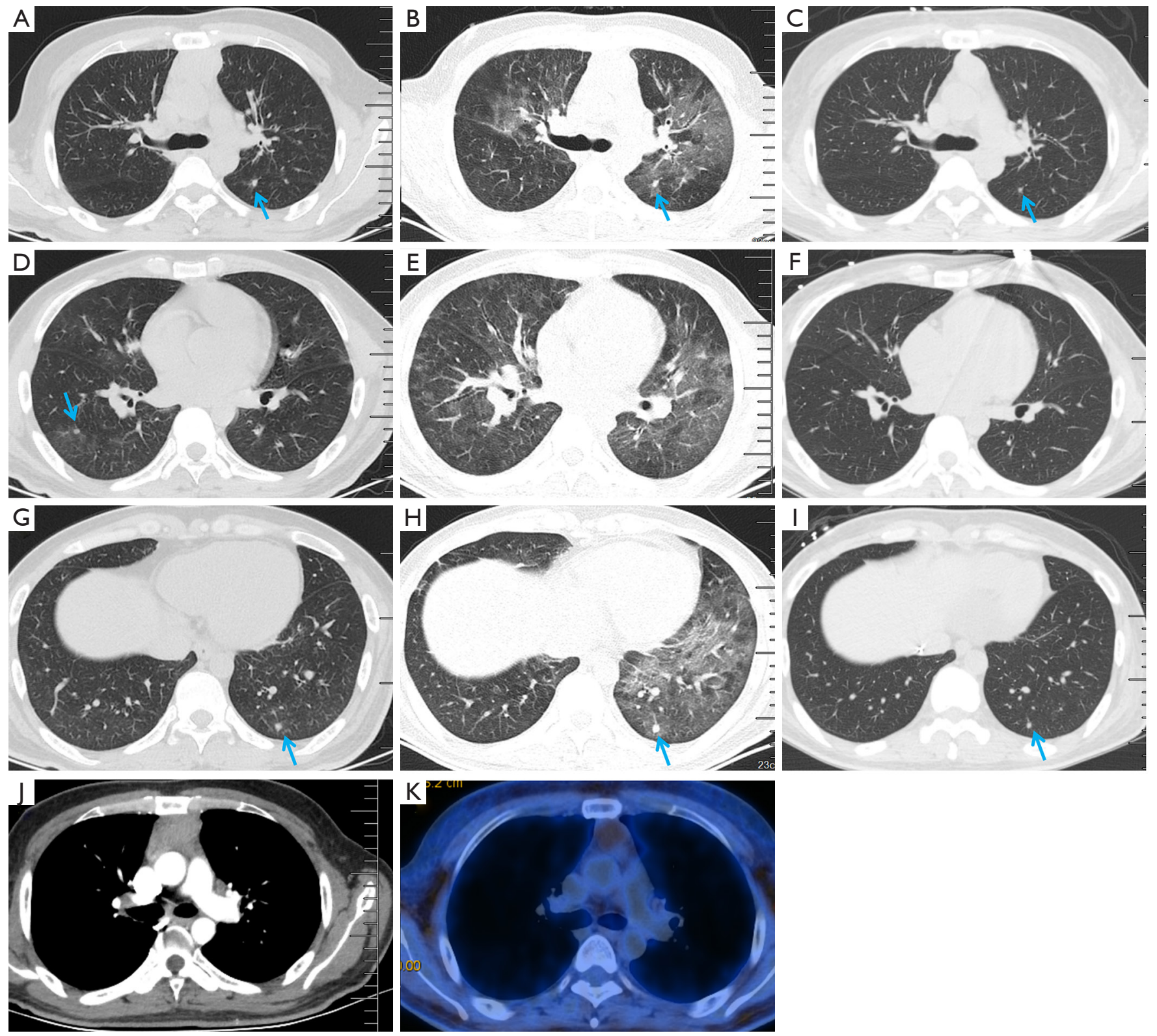

Figure 2 Chest computed tomography (CT) scans. (A,D,G) CT image taken at admission showing scattered ground-glass opacities and small nodules in the lungs. (B,E,H) CT image taken after the thymoma puncture operation showing diffuse ground-glass opacities in the lungs. (C,F,I) CT image taken after hormone and somatostatin combination therapy showing almost complete absorption of the groundglass opacities and a reduction in the size of the lung nodules, with some disappearing. The arrows indicate nodule opacities. (J) Enhanced CT image of the chest taken at admission showing a mediastinal soft tissue mass measuring approximately $4.0 \mathrm{~cm} \times 2.6 \mathrm{~cm}$ in size with unclear margins; the CT value is approximately $45 \mathrm{HU}$, and there is obvious uneven enhancement. (K) Positron emission tomographyCT (PET-CT) image taken after treatment showing a reduction in the size of the mass to approximately $3.3 \mathrm{~cm} \times 2.4 \mathrm{~cm}$. The maximum standardized uptake value $\left(\mathrm{SUV}_{\text {max }}\right)=3.19$. 


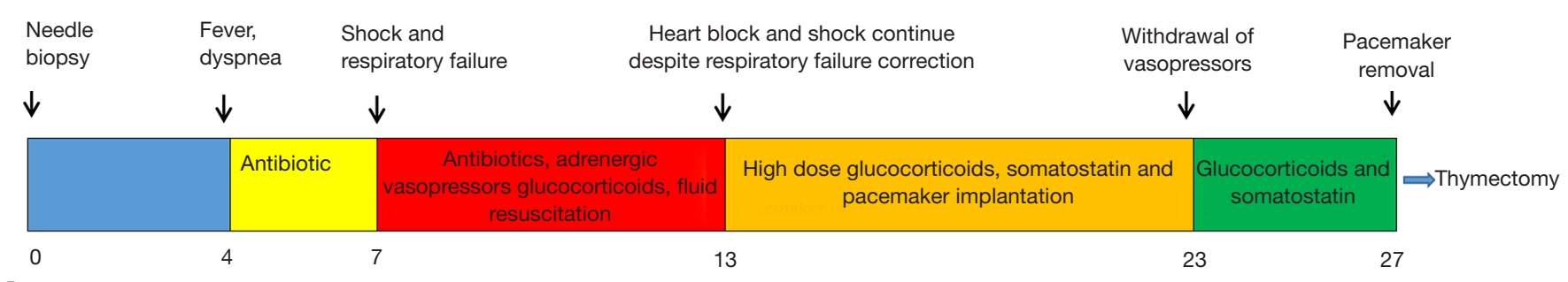

Days

Figure 3 Timeline and duration of each treatment.

Table 1 Changes in vital signs and oxygenation indexes after thymoma biopsy

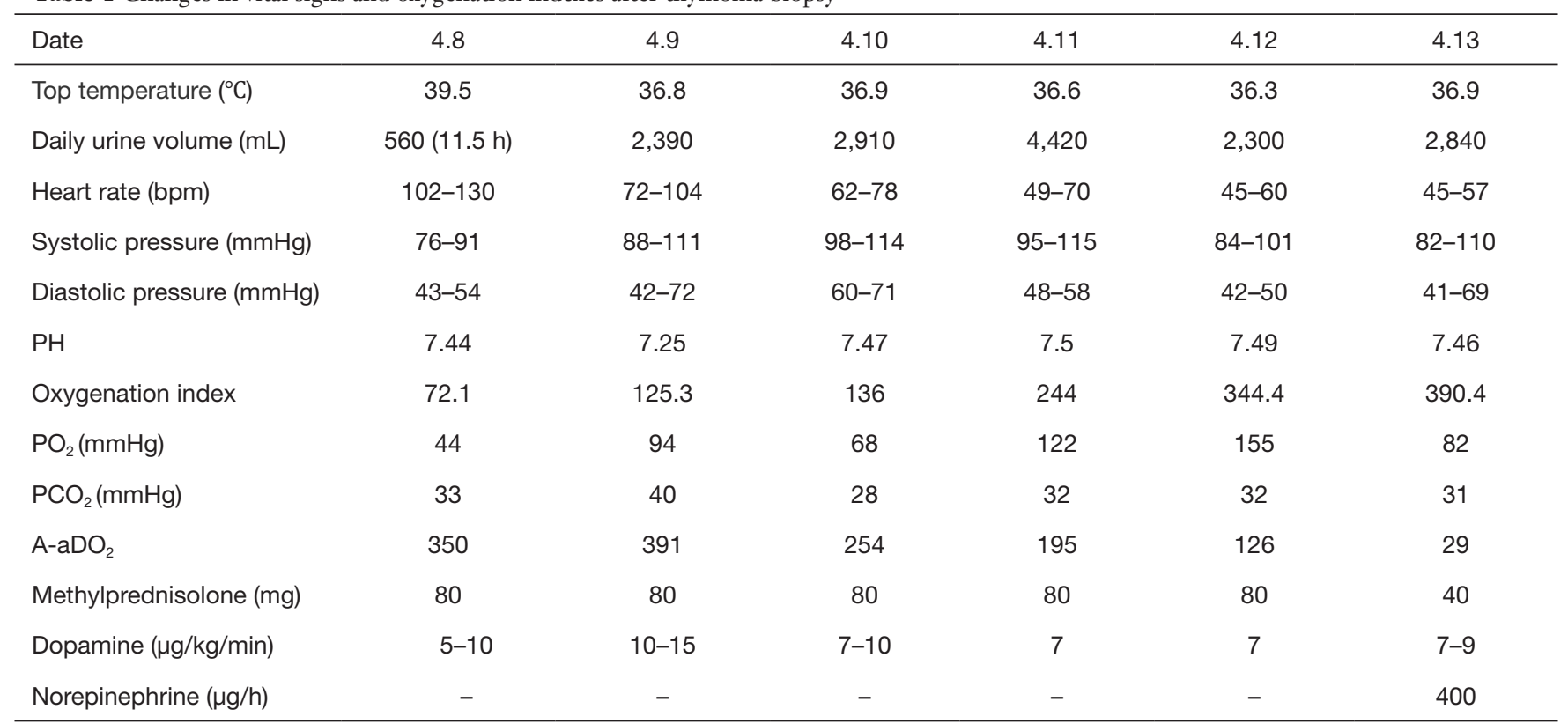

$\mathrm{PH}$, degree of acid or alkali; $\mathrm{PO}_{2}$, partial pressure of oxygen; $\mathrm{PCO}_{2}$, partial pressure of carbon dioxide; $\mathrm{A}-\mathrm{aDO} \mathrm{O}_{2}$, alveolar-arterial oxygen difference.

tolerance test results indicated hypoglycemia (Table 2).

After treatment with somatostatin plus hydrocortisone (100 mg every 12 hours by intravenous drip $\times 3$ day, followed by $150 \mathrm{mg}$ every day by intravenous drip $\times 4$ day, then 100 $m g$ every day by intravenous drip $\times 3$ day, with a gradual change to oral hydrocortisone), the patient's hypotension was corrected and his heart rate stabilized. After 10 days, adrenergic drugs were discontinued, and the temporary pacemaker was removed after 2 weeks. On April 24, wholebody positron emission tomography (PET) indicated that the bilateral ground-glass opacities in the lungs were almost completely absorbed, while the lung lesions (Figure 2) and the thymoma had shrunk in size (Figure 2). No lymph node enlargement was detected in the hilum or mediastinum and no metastasis to the bilateral adrenal glands was found; both the enhanced CT of the upper abdomen (performed on March 28) and PET-CT (performed on April 24) showed bilateral adrenal atrophy (Figure 7). By April 29, the QT interval prolongation and $\mathrm{T}$-wave inversion had been completely restored to normal (Figure 4).

Surgery remains the most commonly used treatment for thymic tumors and has the greatest chance of achieving cure. Therefore, on June 2, the patient underwent transsternal extended thymectomy and lymph node dissection at the Cancer Hospital of China Medical University. No intraoperative pathological examination was performed. Considering that the pulmonary nodules had shrunk markedly, no lung biopsy was performed. Hydrocortisone was given intravenously at a daily dose of $50 \mathrm{mg} \times 3$ days and $100 \mathrm{mg} \times 1$ day before surgery, $100 \mathrm{mg}$ 


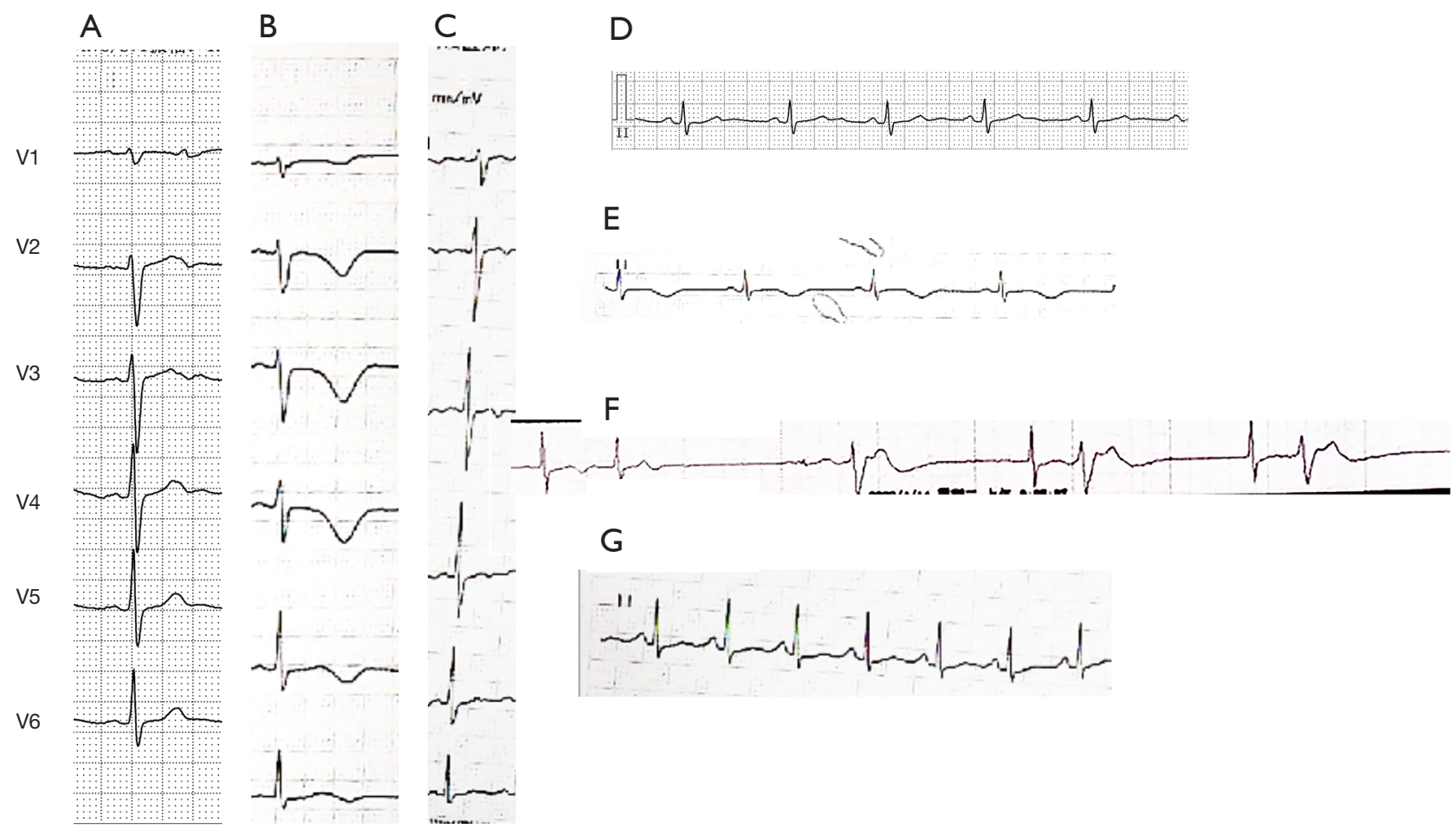

Figure 4 Electrocardiographic findings. (A,D) At admission (March 25, 2020), the patient's heart rate was 66 beats/min, the electrocardiogram (ECG) showed sinus rhythm with a normal QT interval and T wave. (B,E) After thymoma puncture (April 12, 2020), the heart rate was 49 beats/min, the ECG showed sinus bradycardia with a prolonged QT interval $(588 \mathrm{~ms})$ and V1-V6 lead T-wave inversion. (F) Severe heart block on April 14, 2020. After a QRS complex that could not be effectively paced, ventricular premature beats and atrioventricular junctional tachycardia occurred. (C,G) After hormone therapy combined with somatostatin treatment, the temporary pacemaker was removed. The patient's heart rate was 116 beats/min, and the prolonged QT interval and T-wave inversion had returned to normal.
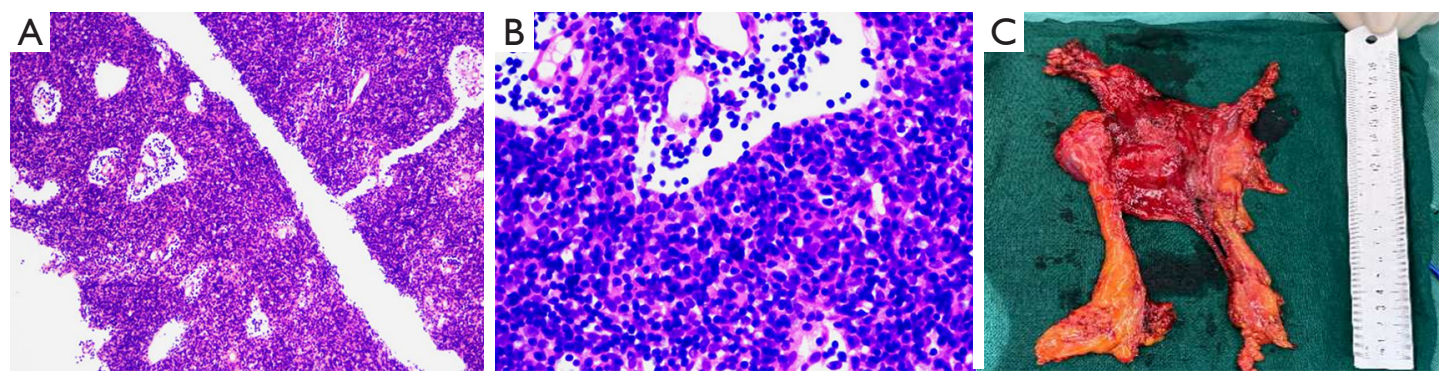

Figure 5 Needle biopsy pathology of the mediastinal mass. (A) Hematoxylin and eosin (HE) staining 100x. (B) HE staining 400x. (C) Complete resection of the thymus. HE staining showing the epithelioid cells to have a sheet-like arrangement. Immunohistochemistry: CK(+); CK19(+); P63(+); CD3(+); CD20(-); CD21(-); Ki-67 (80\%); CD1a(+); TdT(+); CD10(-); CD43(+); CD7(+); CD34(-); In situ hybridization: $\operatorname{EBER}(-)$.

on the operation day, $200 \mathrm{mg} \times 3$ days, $150 \mathrm{mg} \times 2$ days and $50 \mathrm{mg} \times 2$ days after the surgery. The operation was uneventful, and no hypotension or fever occurred; the serum potassium and sodium levels and blood sugar levels were normal afterwards. The pathological examination indicated a Massaoka stage I type B2 thymoma; no abnormal lymph node components were found and some lymphoid tissues showed reactive hyperplasia. After discharge on June 12, 

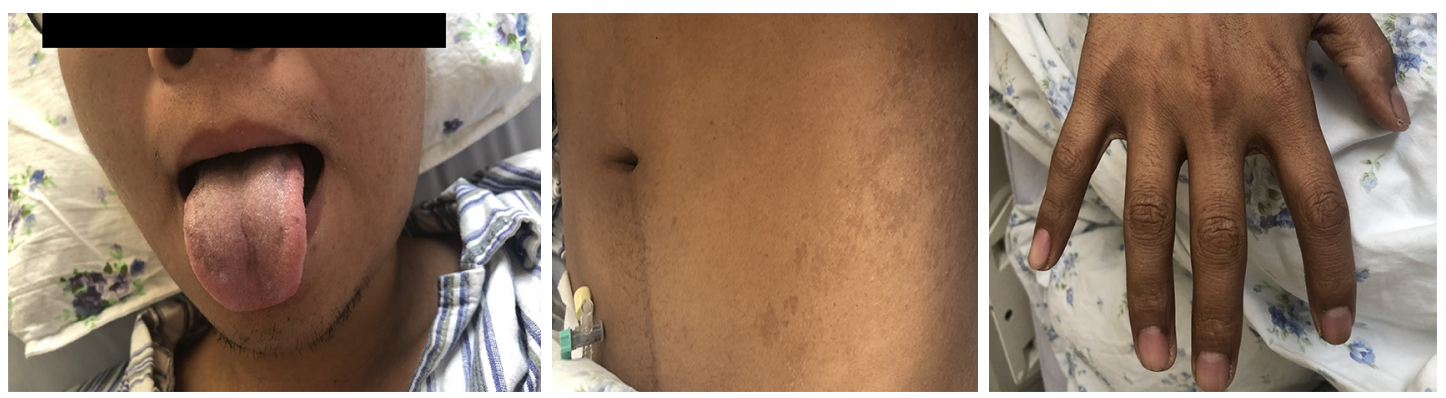

Figure 6 Pigmentation of tongue mucosa, abdominal skin, and fingers.

Table 2 Laboratory indexes after thymoma puncture

\begin{tabular}{|c|c|c|c|c|c|}
\hline \multicolumn{2}{|l|}{$\begin{array}{l}\text { Microbiology } \\
\text { Serum antibody }\end{array}$} & \multicolumn{2}{|c|}{ Endocrine } & \multicolumn{2}{|c|}{ Autoantibody } \\
\hline Influenza virus & $(-)$ & COR $(8 \mathrm{am}) \mu \mathrm{g} / \mathrm{dL}$ & $0.161(6.02-18.4)$ & Anti-TPO IU/mL & $2.03(0-5.61)$ \\
\hline Parainfluenza virus & $(-)$ & ACTH (8am) pg/mL & $1486(7.2-63.3)$ & Anti-Tg IU/mL & $4.39(0-4.11)$ \\
\hline Respiratory syncytial virus & $(-)$ & COR $(4 p m) \mu g / d L$ & $2.59(2.68-10.5)$ & ANA & $1: 160(1: 80)$ \\
\hline Echovirus virus & $(-)$ & COR $(0 \mathrm{am}) \mu \mathrm{g} / \mathrm{dL}$ & 0.45 & Anti-p & $(-)$ \\
\hline Coxsackie virus B & $(-)$ & ACTH (Oam) pg/mL & 14.15 & Anti-SSA/Ro60 & $(-)$ \\
\hline Measles virus & $(-)$ & FT3 pmol/L & $1.59(2.63-5.71)$ & Anti-SSA/Ro52 & $(-)$ \\
\hline Influenza virus nucleic acids & $(-)$ & FBS mmol/L & $3.74(3.9-6.11)$ & Anti-Jo-1 & $(-)$ \\
\hline Parainfluenza virus nucleic acids & $(-)$ & 1hour PBG mmol/L & $6.46(7.8-11.1)$ & Anti-CENP-B & $(-)$ \\
\hline Peripheral blood & & 2hour PBG mmol/L & $6.67(<7.8)$ & Anti-dsDNA & $(-)$ \\
\hline Epstein-Barr virus DNA & $(-)$ & 3hour PBG mmol/L & $4.62(3.9-6.11)$ & MuSK-Ab & $(-)$ \\
\hline Cytomegalovirus DNA & $(-)$ & Serum sodium $\mathrm{mmol} / \mathrm{L}$ & 137 & $A C h R-A b$ & $(-)$ \\
\hline Tuberculosis spot test & $(-)$ & Serum chloride $\mathrm{mmol} / \mathrm{L}$ & 107 & RyR-Ab & $(-)$ \\
\hline Blood germiculture & $(-)$ & Serum potassium $\mathrm{mmol} / \mathrm{L}$ & 3.85 & Titin-Ab & $(-)$ \\
\hline
\end{tabular}

COR, cortisol; ACTH, adrenocorticotropic hormone; FT3, free triiodothyronine; FT4, free thyroxine; TSH, thyroid-stimulating hormone; PTH, parathyroid hormone; FBS, fasting blood glucose; PBG, postprandial glucose; anti-TPO, antithyroid peroxidase antibody; anti$\mathrm{Tg}$, antithyroglobulin antibody; ANA, antinuclear antibody; anti-P, antiribosomal P protein antibody; anti-Sm, anti-Smith antibody; antiCENP-B, anti centromere protein B antibody; anti-dsDNA, anti-double stranded DNA antibody; MuSK-Ab, muscle-specific kinase antibody; AChR-Ab, acetylcholine receptor antibody; RyR-Ab, ryanodine receptor antibody; titin-Ab, titin antibody. 

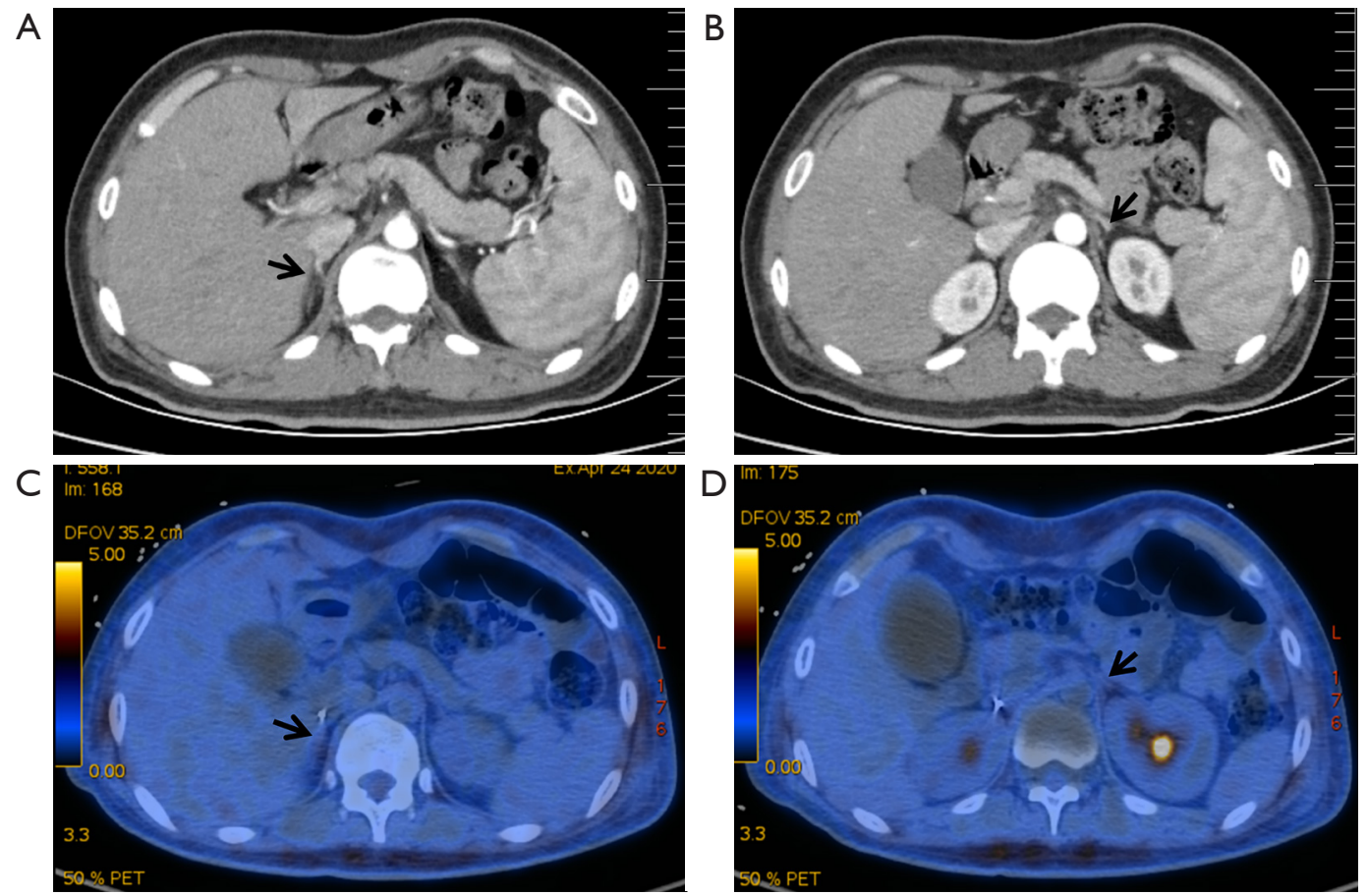

Figure 7 CT findings of the bilateral adrenal glands (A,B) Enhanced CT image of the upper abdomen taken on March 28: (A) shows the right adrenal gland and (B) shows the left adrenal gland. (C,D) Positron emission tomography-CT (PET-CT) image taken on April 24: (C) 'c' shows the right adrenal gland and (D) shows the left adrenal gland. Both enhanced CT and PET-CT showed bilateral adrenal atrophy; ${ }^{18} \mathrm{~F}$-fluorodeoxyglucose $\left({ }^{18} \mathrm{~F}\right.$-FDG) uptake in the bilateral adrenal glands was normal.

2020, the patient continued to take hydrocortisone $(20 \mathrm{mg})$ once daily in the morning, and dexamethasone $(0.5625 \mathrm{mg})$ once daily in the evening orally. Whole-exome sequencing of the resected thymic tissue did not reveal any mutations in the autoimmune regulator gene (AIRE) or other genes. Cortisol $(8 \mathrm{am})(<0.054 \mu \mathrm{g} / \mathrm{dL})$ and ACTH $(8 \mathrm{am})$ $(312.8 \mathrm{pg} / \mathrm{mL})$ were still obviously abnormal at 1 month post surgery. The patient was very satisfied with the treatment.

All procedures performed involving human participants were in accordance with the ethical standards of the institutional and/or national research committee(s) and with the Helsinki Declaration (as revised in 2013). Written informed consent was obtained from the patient for publication of this case report and any accompanying images.

\section{Discussion}

Thymomas are often complicated by paraneoplastic syndromes unrelated to local tumor invasion and metastasis. Paraneoplastic syndromes are autoimmune diseases in which autoantibodies against skeletal muscle, the nervous system, and glands are frequently detected, with myasthenia gravis being the most common of such disorders (8). Fewer than $10 \%$ of patients with thymoma have other autoimmune diseases such as systemic lupus erythematosus, autoimmune thyroid disease, ulcerative colitis, or autoimmune hemolytic anemia (9-12). Moreover, some cases have been considered coincidental, due to the extremely low number of such cases that have been reported (13). One such example is thymoma complicated with autoimmune Addison's disease, of which only 6 cases have been reported in the Englishlanguage literature to date (1-6). Of these 6 cases, all had 2 concomitant autoimmune diseases, none of which included concomitant interstitial lung disease.

In the case reported herein, fatigue, weight loss, and hyperpigmentation of the tongue, mucous membranes, and skin, as well as significantly decreased cortisol and increased adrenocorticotropic hormone levels, clearly indicated Addison's disease. The causes of adrenal insufficiency include autoimmunity, adrenal hemorrhage or infarction, infection (including tuberculosis, human immunodeficiency virus, and disseminated fungal infection), metastasis, 
congenital adrenal hypoplasia, and amyloidosis (14). In the early stages of the disease, our patient had no fever or other signs of infection. Furthermore, whole-body PET/ CT did not indicate adrenal metastasis or infection, the T-SPOT tuberculosis test and HIV test was negative, and the changes observed on lung imaging were inconsistent with tuberculosis. Thus, autoimmune Addison's disease as a paraneoplastic syndrome secondary to thymoma was considered. Due to the limitations of our laboratory conditions, we did not perform anti-adrenal cortex antibody testing, which is a limitation of this report, and wholeexome sequencing yielded negative results.

In patients with long-term untreated Addison's disease, stress can induce adrenal crisis with refractory hypotension and severe arrhythmia. In some cases, the first manifestation is cardiac arrest or congestive heart failure, which can be life-threatening if treatment is not received immediately. The electrocardiographic presentation includes a prolonged QT interval, T-wave inversion, and junctional escape rhythm, which eventually develops into ventricular tachycardia, ventricular fibrillation, and even cardiac arrest. In some cases, in the literature, left ventricular hypertrophy and impaired mobility appeared simultaneously, but all such cases showed improvement after hormone replacement therapy (15-19). Our patient developed fever and shock after needle biopsy. However, after methylprednisolone treatment, the fever subsided rapidly, although the hypotension remained, and the bradycardia gradually progressed. The electrocardiographic performance was almost the same as those reported in the literature. Due to the timely diagnosis and increased hormone dose, the patient avoided a more severe arrhythmia. Thymoma complicated with myocarditis can also cause heart block and ventricular tachycardia. It has been reported that patients have died despite the use of pacemakers and high-dose steroid therapy $(20,21)$. However, van Haelst (22) reported a case of myocarditis occurring 2 weeks after thymectomy; the myocarditis manifested as congestive heart failure and polymorphic ventricular arrhythmias. These conditions were ameliorated by high-dose steroid pulse therapy, the use of an implantable cardioverter defibrillator, plasmapheresis, and immunosuppressive therapy. This suggests that thymoma-associated arrhythmia with myocarditis is more dangerous, and steroid therapy may not be effective in this case. The patient reported herein showed normal levels of cardiac enzymes; further, no evidence of myocarditis was found, the response to steroid therapy was good, and the heart block was more likely to be secondary to adrenal crisis. It is unclear whether thymomas are associated with other paraneoplastic syndromes that affect cardiac conduction. Because endocrine testing confirmed Addison's disease, we hypothesized that the needle biopsy may have induced the patient's adrenal crisis. Treatment with hydrocortisone, somatostatin, and temporary pacemaker implantation successfully restored sinus rhythm and corrected hypotension.

Patients with thymoma can develop vasoplegic shock (high cardiac output with extremely low vascular resistance) within 10 minutes of tumor resection. Although fluid supplementation and adrenergic blood pressure drugs are ineffective, shock can be corrected within 10 minutes after the administration of a somatostatin analog (octreotide) and diphenhydramine (23). The decrease in blood pressure may be associated with vasoactive substances produced by the thymoma, such as histamine, bradykinin, serotonin, prostaglandins, cytokines, or other inflammatory mediators (23), which are released into the blood when tumor tissue is surgically resected, causing an acute decrease in blood pressure. In the present case, adrenal crisis developed after needle biopsy of a thymoma. Glucocorticoid replacement therapy was essential, and the patient's blood pressure increased significantly after the addition of somatostatin without changing other drugs. Chest imaging after treatment revealed shrinkage of the thymoma. According to a previous study, somatostatin plus glucocorticoids is a secondline treatment option for inoperable thymic tumors (24), suggesting its effectiveness against thymoma and its potential value in the treatment of thymomas complicated by adrenal crisis.

The current patient had interstitial lung disease prior to undergoing thymoma needle biopsy. Lung imaging revealed ground-glass opacities and small nodules. Similar lung imaging changes have been reported in thymoma complicated with Good's syndrome and interstitial lung disease, in which lung biopsy pathology indicated granulomatous-lymphocytic interstitial lung disease $(25,26)$. Here, the patient's interstitial lung disease progressed rapidly after the thymoma needle biopsy, with diffuse ground-glass opacities in both lungs, accompanied by a high fever and respiratory failure. Unlike refractory hypotension, fever and respiratory failure were rapidly corrected after methylprednisolone administration. Tests were performed to rule out viral infection, the results of which were all negative. Lung CT re-examination indicated significant absorption of the ground-glass opacities in both lungs, and the lung nodules had also shrunk in size; thus, infectious disease is 
unlikely to be an explanation for these lung lesions.

Glucocorticoids have rapid and good efficacy in patients with autoimmune Addison's disease. When considering lung diseases, we did not exclude thymoma-related autoimmune interstitial lung disease. There have been few studies on the autoantibody profiles of thymoma with lung disease. A previous study found that $46.2 \%$ of patients with thymoma and lung disease have antibodies against lung-specific bactericidal/permeability-increasing foldcontaining B1 and/or potassium channel regulator (27). The immunological mechanism underlying the presence of lung autoantibodies has yet to be illuminated. Mutations in the AIRE in thymic epithelial cells may be a potential mechanism, as this results in impaired central tolerance and escape of peripheral autoreactive $\mathrm{CD}^{+} \mathrm{T}$ lymphocytes (28), which induces the breakdown of B-lymphocyte immune tolerance (29). However, no AIRE mutation was found in the whole genome exon of thymic tissue from our patient; therefore, other unknown immune factors may exist.

\section{Conclusions}

Thymoma is often complicated by paraneoplastic syndromes, and the occurrence of 2 concomitant autoimmune diseases is common. To our knowledge, this is the first report of thymoma with concomitant autoimmune Addison's disease and interstitial lung disease which deteriorated rapidly after needle biopsy. The clinical manifestations of Addison's disease and interstitial lung disease are nonspecific and can be easily overlooked; however, it can induce adrenal crisis in the postoperative period, leading to life-threatening shock, arrhythmias, and acute respiratory failure. Clinicians should be aware of these phenomena and consider proactive glucocorticoid replacement therapy to preserve the lives of such patients. Moreover, shock can be markedly ameliorated by combining glucocorticoid treatment with somatostatin. Furthermore, additional research regarding the aforementioned paraneoplastic syndromes is required to determine the potential of diagnostic testing to serve as a predictor for medical decompensation and to establish the mechanisms underlying the effects of current and future therapies.

\section{Acknowledgments}

The authors greatly appreciate Shumei Ma, Professor of Cardiovascular Medicine for her helpful contribution in curing the patient.
Funding: This research was supported by the National Key Technology R\&D Program Foundation (No. 2018YFC1311901) and the National Natural Science Foundation of China (No. 82073286). Ethical approval was obtained from the Shengjing Hospital of China Medical University Ethics Committee (2021PS052K).

\section{Footnote}

Reporting Checklist: The authors have completed the CARE reporting checklist. Available at https://dx.doi. org/10.21037/tlcr-21-195

Peer Review File: Available at https://dx.doi.org/10.21037/ tlcr-21-195

Conflicts of Interest: All authors have completed the ICMJE uniform disclosure form (available at https://dx.doi. org/10.21037/tlcr-21-195). The authors have no conflicts of interest to declare.

Ethical Statement: The authors are accountable for all aspects of the work in ensuring that questions related to the accuracy or integrity of any part of the work are appropriately investigated and resolved. All procedures performed involving human participants were in accordance with the ethical standards of the institutional and/or national research committee(s) and with the Helsinki Declaration (as revised in 2013). Written informed consent was obtained from the patient for publication of this case report and any accompanying images.

Open Access Statement: This is an Open Access article distributed in accordance with the Creative Commons Attribution-NonCommercial-NoDerivs 4.0 International License (CC BY-NC-ND 4.0), which permits the noncommercial replication and distribution of the article with the strict proviso that no changes or edits are made and the original work is properly cited (including links to both the formal publication through the relevant DOI and the license). See: https://creativecommons.org/licenses/by-nc-nd/4.0/.

\section{References}

1. Boulet P, Passouant P, Mirouze J, et al. Myasthenia with Addison's disease. Phreno-pulmonary metastatic thymoma. Ann Endocrinol (Paris) 1959;20:740-7.

2. Inaba $\mathrm{H}$, Ariyasu $\mathrm{H}$, Iwakura $\mathrm{H}$, et al. Autoimmune 
polyglandular syndrome type 2 and autoimmune hepatitis with thymoma-associated myasthenia gravis: case report. BMC Endocr Disord 2020;20:47.

3. LeGolvan DP, Abell MR. Thymomas. Cancer 1977;39:2142-57.

4. Morita H, Hirota T, Mune T, et al. Paraneoplastic neurologic syndrome and autoimmune Addison disease in a patient with thymoma. Am J Med Sci 2005;329:48-51.

5. Salyer WR, Eggleston JC. Thymoma: a clinical and pathological study of 65 cases. Cancer 1976;37:229-49.

6. Seker M, Gozu HI, Oven Ustaalioğlu BB, et al. Myasthenia gravis and autoimmune Addison disease in a patient with thymoma. Clin Lung Cancer 2009;10:367-70.

7. Betterle C, Dal Pra C, Mantero F,et al. Autoimmune adrenal insufficiency and autoimmune polyendocrine syndromes: autoantibodies, autoantigens, and their applicability in diagnosis and disease prediction. Endocr Rev 2002;23:327-64.

8. Vernino S, Cheshire WP, Lennon VA. Myasthenia gravis with autoimmune autonomic neuropathy. Auton Neurosci 2001;88:187-92.

9. Ben-Shahar M, Rosenblatt E, Green J, et al. Malignant thymoma associated with progressive systemic sclerosis. Am J Med Sci 1987;294:262-7.

10. Souadjian JV, Enriquez P, Silverstein MN, et al. The spectrum of diseases associated with thymoma. Coincidence or syndrome? Arch Intern Med 1974;134:374-9.

11. Thomas CR, Wright CD, Loehrer PJ. Thymoma: state of the art. J Clin Oncol 1999;17:2280-9.

12. Tuncer Elmaci N, Ratip S, Ince-Günal D, et al. Myasthenia gravis with thymoma and autoimmune haemolytic anaemia. A case report. Neurol Sci 2003;24:34-6.

13. Rosenow EC 3rd, Hurley BT. Disorders of the thymus. A review. Arch Intern Med 1984;144:763-70.

14. Ten S, New M, Maclaren N. Clinical review 130: Addison's disease 2001. J Clin Endocrinol Metab 2001;86:2909-22.

15. Eto K, Koga T, Sakamoto A, et al. Adult reversible cardiomyopathy with pituitary adrenal insufficiency caused by empty sella--a case report. Angiology 2000;51:319-23.

16. Iga K, Hori K, Gen H. Deep negative T waves associated with reversible left ventricular dysfunction in acute adrenal crisis. Heart and vessels 1992;7:107-11.

17. Izumi C, Inoko M, Kitaguchi S, et al. Polymorphic ventricular tachycardia in a patient with adrenal insufficiency and hypothyroidism. Jpn Circ J 1998;62:543-5.

18. Kanamori K, Yamashita R, Tsutsui K, et al. Long QT syndrome associated with adrenal insufficiency in a patient with isolated adrenocorticotropic hormone deficiency.
Intern Med 2014;53:2329-31.

19. Nishizawa S, Nakamura T, Hamaoka T, et al. Lethal arrhythmia and corticosteroid insufficiency. Am J Emerg Med 2009;27:1167.e1-3.

20. de Jongste MJ, Oosterhuis HJ, Lie KI. Intractable ventricular tachycardia in a patient with giant cell myocarditis, thymoma and myasthenia gravis. Int J Cardiol 1986;13:374-8.

21. Pentz WH. Advanced heart block as a manifestation of a paraneoplastic syndrome from malignant thymoma. Chest 1999;116:1135-6.

22. van Haelst PL, Brügemann J, Diercks GF, et al. Serial right ventricular endomyocardial biopsy in rapid-onset severe heart failure due to giant cell myocarditis. Cardiovasc Pathol 2006;15:228-30.

23. Williams WH 3rd, Browne RC, Bui TP, et al. Case report on intravenous octreotide for the treatment of intraoperative vasoplegia following thymoma resection. SAGE Open Med Case Rep 2019;7:2050313×19827744.

24. Loehrer PJ Sr, Wang W, Johnson DH, et al. Octreotide alone or with prednisone in patients with advanced thymoma and thymic carcinoma: an Eastern Cooperative Oncology Group Phase II Trial. J Clin Oncol 2004;22:293-9.

25. Jensen ML, Bendstrup E, Hilberg O. Granulomatouslymphocytic interstitial lung disease and recurrent sinopulmonary infections in a patient with Good's syndrome. BMJ Case Rep 2015;2015:bcr2014205635.

26. Narahari NK, Gongati PK, Uppin SG, et al. A 66-YearOld Man With Mediastinal Mass and Dyspnea. Chest 2016;150:e109-15.

27. Ferré EMN, Break TJ, Burbelo PD, et al. Lymphocytedriven regional immunopathology in pneumonitis caused by impaired central immune tolerance. Sci Transl Med 2019;11:eaav5597.

28. Mathis D, Benoist C. Aire. Annu Rev Immunol 2009;27:287-312.

29. Sng J, Ayoglu B, Chen JW, et al. AIRE expression controls the peripheral selection of autoreactive B cells. Sci Immunol 2019;4:eaav6778.

Cite this article as: Yan H, Qi Q, Zhang K, He B, Chu G, Wang G, Wen H, Zhao Y, Liu H, Zhao L, Chen Y. Adrenal crisis and acute exacerbation of interstitial lung disease after thymoma needle biopsy: a case report and literature review. Transl Lung Cancer Res 2021;10(7):3292-3302. doi: 10.21037/ tlcr-21-195 